alkohol unter denselben Umständen Jodbutyl giebt, das erst unter Mitwirkung der Wärme auf essigsaures Silber einwirkt und dabei, ohne dafs eine Spur Butylen entsteht, das von Wurtz dargestellte essigsaure Butyl bildet. Wird das Butylenhydrat in geschlossenen Röhren 4 bis 5 Stunden lang auf 240 bis $250^{\circ}$ erhitzt, so spaltet es sich zu Wasser und Butylen; indem ich die Röhre in einer Kältemischung öffnete, konnte ich das Butylen isoliren, für welches ich die Eigenschaften constatirt habe.

Das Butylenhydrat gab bei der Analyse 64,33 pC. C, $13,90 \mathrm{H}$ und 21,770 .

Man ersieht aus dem Vorhergehenden, dafs das jodwasserstoffsaure Butylen und das Butylenhydrat gegenüber den entsprechenden, von dem durch Gährung entstandenen Butylalkobol sich ableitenden Verbindungen ebensolche Beziehungen der Isomerie zeigen, wie sie $W$ urtz für das jodwasserstoffsaure Amylen und das Amylenhydrat einerseits und die entsprechenden, von dem durch Gährung entstandenen Amylalkohol sich ableitenden Verbindungen andererseits nachgewiesen hat.

Diese Versuche sind in dem Untersuchungs-Laboratorium der Faculté des Sciences zu Paris ausgeführt worden.

\title{
Ueber den Cyanphosphor;
}

von G. Wehrhane und H. Hubner.

Da hauptsächlich nur die Verbindungen des Cyans mit den ein- und zweiäquivalentigen Grundstoffen genau erforscht sind, so hielten wir's für geeignet, die Darstellung und Unter- 
suchung einiger Verbindungen des Cyans mit mehräquivalentigen Grundstoffen vorzunehmen.

Ueber derartige Verbindungen sind jetzt nur sehr dürftige Beobachtungen bekannt. Millon*) will eine Verbindung des Stickstoffs mit dem Cyan bei der Einwirkung von Chlorstickstoff auf eine Cyankaliumlösung beohachtet haben; doch bleibt, wie Berzelius bemerkt, das Vorhandensein dieser Verbindung nach diesem Versuch höchst zweifelhaft. Wir haben den Cyanstickstoff bis jetzt vergeblich aus dem gelben Silbercyanamid ( $\mathrm{CNAg} \mathbf{A g}_{2} \mathbf{N}$ ) mit einer ätherischen Jodcyanlösung zu erhalten gesucht. Selbst bei sehr langem und hohem Erhitzen scheinen diese Stoffe nicht aufeinander zu wirken.

Wenig gründlicher wie die von dem Cyanstickstoff ist unsere Kenutnifs des Cyanphosphors, denn aufser einer Mittheilung von Cenedella**), in der dieser den Cyanphosphor als einen weifsen Beschlug beschreibt, welchen er zuweilen bei der Einwirkung von Phosphor auf Cyanquecksilber erhalten hat, sind uns nur noch folgende Andeutungen über denselben bekannt. Erstens hatte H. Davy**) schon eine Verbindung des Cyans mit Phosphor zu erhalten geglaubt, als er Cyanquecksilber mit Phosphor zusammen erhitzte, und zweitens fand $\mathrm{Ke} \mathrm{mp}+$ ), dafs das zu einer Flüssigkeit verdichtete Cyan Phosphor zu lösen vermag; er vermuthet hier das Entstehen einer Verbindung. Drittens hat Stas, wie er uns mittheilte, schon vor sehr langer Zeit krystallisirten Cyanphosphor bei einem Vorversuch erhalten, ohne jedoch die

*) Ann. chim. phys. LXIX, 76 und Marchand, J. pr. Chem. XIX, 5 .

**) J. pharm. XXI, 683 oder Ann. Pharm. XVIII, 70.

***) Gilb. Ann. LIV, 384.

†) Givelin's Handb., 4. Bd., 508. 
Wehrhane u. Bübner, über den Cyanphosphor. 279

Verbindung, ebensowenig wie die oben genannten Chemiker, zu analysiren und zu untersuchen oder nur diese Beobachtung zu veröffentlichen.

Wir geben hier in Anschlufs an eine frühere Mittheilung *) die ausführliche Beschreibung des Cyanphosphors, seine Darstellung und die Bestimmung seiner Zusammensetzung, und glauben ferner den Nachweis liefern zu können, dafs es höchst wahrscheinlich nur Eine Verbindung zwischen Cyan und Phosphor giebt.

Durstellung des Cyanphosphiors. - 20 bis 25 Grm. vollkommen trockenes Cyansilber werden in einer starken Röhre unter Abkühlung vollständig mit Phosphorchlorür befeuchtet und die Röhre immer noch unter guter Abkühlung, da die Umsetzung oft sehr bald unter starker Wärmeentwickelung eintrith, zugeschmolzen. Ein Zusatz von Chloroform oder Aether zum Phosphorchlorür verlangsamt die Einwirkung und verhindert leicht eine vollständige Umsetzung. Nach 6 bis 8 stündigem Erhitzen der Röhre auf 130 bis $140^{\circ}$ wird sie geöflnet, mit einem Kühler verbunden und das überschüssige Phosphorchlorür durch schwache Erwärmung vertrieben. Dann wird der erkaltete trockene Röhreninhalt in eine kleine Retorte durch die Eingufsöffnung gebracht und die Retorte mit aufgerichtetem Hals bis an diese Eingufsõfnung in ein Bad getaucht und auf 130 bis $140^{\prime \prime}$, höchstens $180^{\circ}$ erhitzt. Zur schnelleren Entfernung des letzten Antheils von Phosphorchlorür und leichteren Verflüchtigung des Cyanphosphors kann man einen langsamen Strom trockener Kohlensäure durch die Retorte leiten. Tritt keine Vermehrung der weifsen langen Nadeln und dicken Tafeln des Cyanphosphors im Retortenhals mehr ein, so verschliefst man diesen luftdicht und läfst erkalten. Dann werden die Krystalle mit einen an Ende ungebogenen

*) Diese Annulen CXXVIII, 254. 
Glasstab herausgenommen. Es ist hierbei besonders darauf zu achten, dafs der Cyanphosphor nicht mehr warm ist, da er sonst an der Luft verbrennt. Man erhält so $\mathbf{4 , 5}$ bis $\mathbf{4 , 8}$ Grm. Cyanphosphor, die Berechnung verlangt 5,4 Grm. Nicht vortheilhaft ist es, sehr grofse Mengen Cyansilber und Phosphorchlorür in eine Röhre zu bringen, da sonst die Durchwärmung nicht gleichmälsig erfolgen kann.

Bestimmung der Zusammensetzung. - Zur Phosphorbestimmung wurden die Krystalle unmittelbar aus dem Retortenhals in ein gewogenes Röhrchen gebracht und gut verschlossen gewogen; darauf das Röhrchen offen in ein hohes, nur wenig mit Wasser gefülltes Becherglas gebracht und durch Erwärmung Wasserdämpfe gebildet, welche den Cyanphosphor allmälig zersetzten, darauf das Röhrchen und Becherglas mit starker Salpetersäure ausgewaschen und das Waschwasser mit Ammoniak und Salmiak versetzt und mit schwefelsaurem Magnesium gefüllt.

Oder das Röhrchen mit dem Cyanphosphor wurde in eine mit Salpetersäure wenig gefüllte Röhre gebracht, diese dann zugeschmolzen und geschüttelt und erhitzt, um den Cyanphosphor zu zersetzen. Auf diesen beiden Wegen wurden die folgenden Bestimmungen mit Cyanphosphor verschiedener Darstellungen ausgeführt :

1. 0,1820 Grm. Cyanphosphor gaben 0,18825 phosphorsaure Magnesia, d. l. 28,86 pC. P.

2. 0,3494 Grm. Cyanphosplior gaben 0,3515 phosphorsaure Magnesia, d. h. 28,04 pC. P.

3. 0,6045 Grm. Cyanphosphor gaben 0,6335 phosphorsaure Magnesia, d. h. 27,92 pC. P.

Zur Cyanbestimmung konnte der Cyanphosphor in derselben Weise wie bei der Phosphorbestimmung in einem Röhrchen gewogen und in eine mit Salpetersäure und salpetersaurem Silber gefüllte Röhre gebracht werden, und 
zwar wurde nur so viel Flüssigkeit angewandt, dafs das Röhrchen, wenn es auch nicht schwamm, doch nicht ganz unterging; aufserdem war es mit einem abstehenden hand versehen, um das Heraufkriechen der Flüssigkeit an seinen Wänden zu verhindern. Nach dem Zuschmelzen der äufseren Röhre wurde sie vorsichtig geneigt und geschüttelt, dann wieder geöffnet und das Cyansilber auf ein gewogenes Filter gebracht:

1. $0,6045 \mathrm{Grm}$. Cyanphosphor gaben $2,1955 \mathrm{Ag} \in \mathrm{N}$, d. h. 70,5 pC. $\mathrm{GN}$.

2. $0,5714 \mathrm{Grm}$. Cyanphosphor gaben $2,1010 \mathrm{Ag} \in \mathrm{N}$, d. b. $71,33 \mathrm{pC}$. $\mathrm{GN}$. $\mathbf{P}(\mathrm{GN})_{3}$ verlangt :

\begin{tabular}{|c|c|c|}
\hline \multicolumn{3}{|c|}{ berechnet } \\
\hline $\mathbf{P}$ & 31 & 28,4 \\
\hline$(\mathbf{G N})_{\mathrm{s}}$ & 78 & 71,5 \\
\hline & 109 & 99,9 . \\
\hline
\end{tabular}

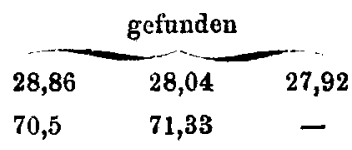

Diese Bestimmungen sind nicht ganz leicht auszuführen, wegen der grofsen Zersetzbarkeit des Cyanphosphors; besonders hält es schwer, bei der Cyanbestimmung richtige Zahlen zu erhalten, wie diel's bei der immerhin leichten Zersetzbarkeit des Cyansilbers nicht zu verwundern ist. Wir erhielten nur richtige Werthe bei Anwendung starker Salpetersäure, un durch die phosphorige Säure abgeschiedenes Silber leicht in der Kälte, ohne Cyansilber zu zersetzen, aufzulōsen. Ohne diese Vorsicht oder ohne Vermeidung von Wärme ergaben sich bei drei Bestimmungen folgende Werthe : $73,4,73,5,70,0$ pC. GN.

Noch ungünstiger fallen die Zahlen aus, versucht man den Cyanphosphor in einem verschlossenen Gefäfs mit Wasser zu zersetzen und die Blausäure abzutreiben und zu fällen. Es scheint fast, dafs die Blausäure in Gemeinschaft mit phosphoriger Säure Wasser zerlegt und sich verändert.

Eigenschaften des Cyanphosphors. - Die weísen Nadeln wie die derben Tafeln des Cyanphosphors entzünden sich 
schon bei Berührung mit einem erwärmlen Glasstab und brennen an der Luft mit hellem Licht. Läfst man die Krystalle an gewöhnlicher feuchter Luft liegen, so zerfallen sie allmälig unter Abscheidung von Phosphor und phosphoriger Sãure und Verdunstung von Blausäure; auch die gröfseren Tafeln halten sich nur 10 bis 15 Minuten unverändert, eine zu kurze Zeit, um sie zu messen. In Chloroform, Aether, Schwefelkohlenstoff und Phosphorchlorür ist der Cyanphosphor nur sehr wenig löslich ; erst bei $200^{\circ}$, bei seinem Schmelzpunkt, scheint er in diesen Flüssigkeiten leicht löslich zu sein. Zur Bestimmung des Schmelzpunktes wurden die Krystalle möglichst schnell in die hierzu gebräuchlichen dünnwandigen Röhrchen gebracht, welche vorher mit trockener Kohlensäure angefüllt worden waren. Vollständig geschmolzen waren sie bei 200 bis $203^{\circ}$; der Siedepunkt liegt nur wenige Grade höher. Der geschmolzene Cyanphosphor hält sich bei niedriger Wärme lange Zeit flüssig, erstarrt aber bei der geringsten Berührung mit einem spitzen Gegenstand.

Mit Wasser zersetzt sich der Cyanphosphor lebhaft in Blausäure, die leicht als Berlinerblau nachzuweisen war, und in phosphorige Säure, welche durch Quecksilberchlorid erkannt wurde.

Man durfte sich der Hoffnung hingeben, in dem Cyanphosphor die Verbindung gefunden zu haben, mittelst der es gelingen würde, in ganz entsprechender Weise, wie durch das Phosphorchlorür das Chlor, so hier das Cyan in die organischen Verbindungen einführen zu können. Zur Prüfung dieser Yoraussetzung wurden einige organische Verbindungen mit Cyanphosphor zusammengebracht. Leider zeigte es sich, dafs der Cyanphosphor doch nur wenig zu diesem Zweck geeignet ist, und zwur aus dem einfachen Grund, weil die organische Cyanverbindung einerseits durchgängig weniger flüchtig und andererseits weit leichter zersetzbar ist, als die 
Wehrhane u. Bübner, über den Cyanphosphor. 283

entsprechende Chlor- oder Brom- oder Jadverbindang, so dafs die bei der Zersetzung des Cyanphosphors mit sauerstoffhaltigen Verbindungen gebildeten organischen Cyanide durch die gleichzeitig entstehende phosphorige Säuro sehr leicht zerstört werden, besonders wenn man die phosphorige Säure und das Cyanid durch Destillation trennen will, bei welcher Gelegenheit meist schon bei $100^{\circ}$ eine stürmische Zersetzung unter Verkohlung und Entwickelung stinkender Gase eintritt. Und doch bleibt die Destillation bis jetzt das cinzige Miltel, um das Cyanid von der phosphorigen Sāure zu trennen. - Bei der Einwirkung des Cyanphosphors anf Alkohole konnte man annehmen, dafs die Zersetzung verschieden verlaufe, je nachdem man auf 3 Atome Alkohol 1 Atom oder 2 Atome Cyanphosphor anwende :

$$
\begin{aligned}
& \text { 1) } \mathrm{P}(\mathbf{G N})_{8}+3 \boldsymbol{G}_{2} \mathrm{H}_{6} \stackrel{\theta}{\stackrel{\theta}{H}}=P\left(\underset{G_{2} \mathrm{H}_{0}}{\stackrel{\theta}{s}}\right)_{3}+3 \in \mathrm{NB} \text { oder } \\
& \text { 2) } P\left(\underset{G_{2} H_{6}}{\theta}\right)_{8}+P(\Theta N)_{8}=P_{8} \Theta_{8}+3 G_{8} B_{6} \in N \text {. }
\end{aligned}
$$

Der Versuch nach Gleichung 1 wurde ausgeführt, indem in eine starke Röhre einmal eine abgewogene Menge Aethylalkohol, ein andermal Amylalkohol gebracht wurde, und dann die entsprechende Menge Cyanphosphor in einem Röhrchen hinzugefügt; darauf wurde die äufsere Rōhre zugeschmolzen und geneigt, um so die heftige Zersetzung ohne Verlust herbeizuführen. Nach dem Oeffnen der Röhre konnte eine grofse Menge wasserfreier Blausảure abgedunstet werden; im Rũckstand blieb dann phosphorigsaurer Aether, der leicht an seinen Eigenschaften und seinem Siedepunkt ( $191^{\circ}$ beim Aethyläther) erkannt werden konnte. Neben dem Geruch der Blausāure bemerkte man bei diesen Versuchen stets noch denselben abschenlich stinkenden Geruch, durch welchen man bei der Cyanăthylbereitung aus Cyankalium und ätherschwefelsaurem Natrium belästigt wird. Ein in gleicher Art ausgeführter Versuch, bei dem auf 2 Atome Cyanphosphor nur 3 Atome 
Alkohol angewandt worden waren, hatte einen gleichen Verlauf, nur trat bei der Destillation eine noch heftigere Zersetzung und Verkohlung ein, aber weder Cyanäthyl noch Propionsäure konnte nach längerem Erhitzen des Röhreninhalts mit Kalilauge nachgewiesen werden. Das aus dem Kaliumsalz erhaltene Baryumsalz hatte folgenden Baryumgebalt :

$0,4895 \mathrm{Grm}$. des Salzes gaben 0,5000 schwefels. Baryt, d. b. $67,08 \mathrm{pC}$. und $6 \mathrm{HBa} \theta_{2}$ verlangt $67,57 \mathrm{pC}$. Baryt.

In ganz gleicher Art wie die Alkohole liefs man Eisessig und entwässerte Valeriansäure auf Cyanphosphor einwirken. Auch hier wurde die Säure und der Cyanphosphor in verschiedenen Verhältnissen zusammengebracht :

1) $\mathrm{P}(\mathrm{GN})_{3}+3 \mathrm{G}_{2} \mathrm{H}_{3} \boldsymbol{\theta}_{\mathrm{H}}^{\Theta}=\mathrm{P}(\stackrel{\theta}{\mathrm{H}})_{3}+3 \mathrm{G}_{2} \mathrm{H}_{8} \theta \cdot \mathrm{GN}$.

2) $2 \mathrm{P}(\mathrm{GN})_{8}+36_{2} \mathrm{H}_{3} \theta_{\mathrm{H}}^{\ominus}=\mathrm{P}_{2} \theta_{\mathrm{g}}+3 \mathrm{G}_{2} \mathrm{H}_{3} \Theta .6 \mathrm{~N}+3 \mathrm{GNH}$.

Der Versuch verlief in beiden Fällen ganz gleichartig, nur erfolgte eine bis zur Verkohlung gehende Zersetzung in 2. Fall besonders schon bei sehr niederer Wärıne, wenn man Essigsäure anwendete. Die Valeriansäure liefert neben etwas Blausäure und phosphoriger Säure eine dritte in Wasser unlösliche Flüssigkeit, die sich daher leicht abscheiden liefs, und welche, nachdem sie gründlich mit kohlensaurem Natrium durchschüttelt worden war, mit Wasser oder leichter Alkalien gekocht in Blausäure und Valeriansäure zerfiel und überhaupt das Verhalten und den Geruch des von D. Cunze und H. Hübner aus Cyansilber und Chlorvaleryl dargestellten Valercyanids zeigte. Hiernach und wegen der Gleichartigkeit des Phosphorchlorürs und Cyanphosphors ist anzunehmen, dafs die Zersetzung glatt stels nur nach der ersten Gleichung verläuft, welche Umsetzung noch dadurch bestätigt wird, dafs man bei derartigen Zersetzungen auch mit Phosphorchlorür nicht eine trockene 
Welirhane u. Hübner, über den Cyanphosphor. 285

Masse wie $\mathbf{P}_{2} \theta_{3}$ erhält, sondern einen zăhen Syrup wie $\mathbf{P} \boldsymbol{\theta}_{3} \mathbf{H}_{3}$. Die grofse Anziehung der phosphorigen Sãure zum Wasser läfst diefs auch erwarten.

Es wurden ferner noch einige kurz zu erwähnende Einwirkungen des Cyanphosphors auf andere organische Verbindungen beobachtet. Die Zersetzung des Chloracetyls und Cyanacetyls mit Cyanphosphor konnte zu merkwürdigen Verbindungen führen, vorausgesetzt, dafs der Sauerstoff dioser Verbindungen sich leichter durch Cyan als durch Chlor aus dem Phosphorchlorür ersetzen lasse, was nicht eben wahrscheinlich war. In der That ging die Zersetzung auch erst bei $100^{\circ}$ vor sich und verlief zu wenig glatt, um verfolgt werden zu können. Trockenes Ammoniak scheint gar keine Einwirkung auf Cyanphosphor auszuüben; erhitzt man den Cyanphosphor gleichzeitig, so verwandelt er sich in eine schwarze krümelige, in Wasser unlösliche Masse.

Bei all diesen Versuchen war man durch die kostbare und mübsame Darstellung des Cyanphosphors sehr behindert; es wurde daher wünschenswerth, eine ergiebigere Quelle für den Cyanphosphor aufzufinden. Zu diesem Zweck versuchten wir zunächst, durch das Einleiten von Chlorcyangas in Phosphordampf Cyanphosphor zu erhalten. Der gelbrothe Beschlag des erkalteten Phosphors zeigte aber nach Verdrängung des Chlorcyans durch Kohlensäure nur Spuren von Salzsäure, Blausäure und phosphoriger Säure. Ebenfalls konnte Cyangas in Phosphordampf geleitet werden, ohne dafs eine Verbindung eintrat. Hierauf suchte man das Cyansilber durch die Cyanverbindungen von Quecksilber, Zink, Kalium, Kupfer und Blei zu ersetzen, aber bein Erhitzen mit Phosphorchlorür auf 150 bis $200^{\circ}$ konnte keine Umsetzung erzielt werden. Endlich versuchten wir, den Cyanphosphor aus wasserfreier Blausäure und Phosphorchlorür darzustellen. Es war nicht ganz unmöglich, dafs sich Salz- 
sãure und Cyanphosphor bilden möchte. Der Versuch lehrte aber, dafs man diese Verbindungen in einer zugeschmolzenen Röhre auf 160 bis $170^{\circ}$ ohne durchgreifende Zersetzung zu erreichen erhitzen kann. Oeffnet man dann die Röhre, so ist kein Druck vorhanden; also hat keine Salzsäurebildung stattgefunden. Ein Theil der Blausãure zersetzt sich hierbei mit einer schwer zu vermeidenden Spur von Wasser in Salmiak und phosphorige Säure und also wahrscheinlich auch Ameisensäure, während ein anderer Theil einen braunen flockigen Absatz bildet.

Einwirkung von Fünffach-Chlorphosphor auf Cyansilber. - In Verhältnifs von 1 Alom Phosphorchlorid (mit Schwefelkohlenstoff als Lõsungsmittel) und 5 Atomen Cyansilber wurden diese Verbindungen 5 bis 6 Stunden in einen zugeschmolzenen Rohr auf 150 bis $160^{\circ}$ erhitzt. Beim Oeffnen der Röhre zeigte sich Druck und der Geruch nach Chlorcyangas, und weifse Krystalle hatten sich in der Glasröhre angesetzt. Daher wurde die Röhre mit einem Kühler verbunden, der Schwefelkohlenstoff abgedunstet und das entweichende Gas in Ammoniak geleitet. Als dann die ammoniakalische Lōsung mit salpetersaurem Silber versetzt wurde, fiel der von Geuther und Beilstein beschriebene gelbe Niederschlag von Cyansilberamid, der ausgewaschen und getrocknet auf Platinblech verpuffte und mit Kali Ammoniak entwickelte. Die weifsen Krystalle zerfielen mit Wasser in phosphorige Säure, die mit Quecksilberchlorid und ammoniakalischer Silberlösung erkannt wurde, und in Blausăure, die am Geruch und durch Berlinerblaubildung nachzuweisen war. Eine wässerige Lōsung der Krystalle mit Ammoniak, Salmiak und schwefelsaurem Magnesium versetzt lieferte selbst nach 2 Tagen keine Trübung von phosphorsaurem Ammoniak-Magnesium. Also ist die Zersetzung folgende :

$$
\mathrm{PCl}_{\mathrm{b}}+4 \mathrm{Ag} \Theta \mathrm{N}=\mathrm{P}(\mathrm{GN})_{\mathrm{s}}+\mathrm{GNCl}+4 \mathrm{AgCl} \text {. }
$$


Wehrhane u. Bübner, über den Cyanphosphor. 287

2 Atome Chlor des Phosphorchlorürs wirken auch hier wie freies Chlor.

Wenn es somit wahrscheinlich ist, dafs es kein $P(G N)_{5}$ giebt, so konnte es noch entsprechend dem $\mathrm{PJ}_{z}$ eine oyanãrmere Phosphorcyanverbindung als $P(G N)_{s}$ geben. Zur Entscheidung dieser Frage wurde eine Lösung von viel Phosphor in Schwefelkohlenstoff mit wenig Cyanphosphor in einer zugeschmolzenen Röhre lange Zeit auf $210^{\omega}$ erhitzt. Hierdurch wurde etwas Cyanphosphor zersetzt und es schied sich eine Krystallkruste an den Wänden ab; diese Kruste herausgenommen, sorgfältig in Schwefelkohlenstoff gewaschen, getrocknet und gewogen, gab folgenden Phosphorgehalt :

$0,1770 \mathrm{Grm}$. der Verbindung gaben $0,2055 \mathrm{Mg}$-Sale, d. b. $32,43 \mathrm{pC}$., und $P_{8}(G N)_{6}$ verlangt 32,29 pC. $P$.

Das Vorhandensein einer solchen Verbindung $P_{y}(G N)_{3}$ wird aber unwahrscheinlich, da erstens immer noch Phosphor den Krystallen beigemengt sein konnte, und zweitens bei zwei anderen Versuchen und Erhitzung auf $230^{\circ}$ kein Phosphorcyanür entstand, wie folgende Bestimmungen zeigen :

1) 0,1565 Grm. der Verbindung gaben 0,1585 Grm. Mg-Balz, d. h. 27,4 pC. P;

2) 0,4730 Grm. gaben 0,4780 Grm. Mg-\$alz, d. h. $28,16 \mathrm{pC}$., und $P(\Theta N)_{2}$ verlangt $28,4 \mathrm{pC} . \mathrm{P}$.

Erhitzt man Phosphor und Cyanphosphor noch höher als $230^{\circ}$, so kann man ein neben rothem Phosphor möglicherweise entstehendes Phosphorcyanür nicht leicht von diesem trennen.

Es ist also anzunehmen, dafs bis jetzt nur Eine Verbindung des Phosphors mit dem Cyan dargestellt werden kana.

Eine Einwirkung von Phosphoroxychlorid auf Cyansilber konnte selbst bei langem Erhitzen dieser Verbindungen auf $180^{\circ}$ nicht beobachtet werden; das Cyansilber blieb scheinbar unverändert, und es liefs sich nur reines Phosphoroxychlorid von ihm abdunsten. 
Es sei hier noch ein Versuch erwähnt, welcher freilich nicht unmittelbar hergehört : Herr Prof. $\mathrm{S} t$ a $S$ war so freundlich, uns mitzutheilen, dafs er vor langer Zeit durch die Einwirkung von Wasser auf ein Gemisch von Phosphorchlorür und wasserfreier Blausäure eine vielleicht cyanhaltige Phosphorsäure erhalten zu haben glaube. Zur Prüfung dieser Beobachtung wurde Phosphorchlorür in ein kleines Becherglas gebracht, wasserfreie Blausäure darüber geschichtet, das Glas in eine Schale mit etwas Wasser gestellt und diese dann unter eine Glocke gebracht. Ziemlich bald war darauf das Wasser in einen Krystallbrei umgewandelt und das Phosphorchlorür mit einer festen Krystallkruste bedeckt. Giefst man die Flüssigkeit aus dem $W$ asserbehälter in einen Kolben und destillirt, so geht erst eine geringe Menge wahrscheinlich noch nicht umgewandelte Blausăure über, darauf eine sehr grofse Menge von Ameisensäure, die leicht mit Quecksilberoxyd an ihrem eigenthümlichen basischen Salz erkannt werden konnte. Im Rūckstand blieb phosphorige Säure. Die Krystallmasse, sowohl aufserhalb als innerhalb des Gefăfses, bestand zum grōfsten Theil aus Salmiak und phosphorigsaurem Ammoniak. Es konnte niemals in den sorgfältig mit Alkohol gewaschenen Krystallen Blausäure nachgewiesen werden. Es scheint uns hiernach unwahrscheinlich, dafs bei dieser Zersetzung die Bildung einer cyanhaltigen Phosphorverbindung stattindet.

Um die Einwirkung von Phosphorchlorür anf Schwefelcyansilber zu untersuchen, erhitzte man 22,5 Grm. (3 Aeq.) Rhodansilber mit $6 \mathrm{Grm}$. (1 Aeq.) Phosphorchlorür in einer verschlossenen Röhre. Das Rhodansilber mufste mit Aether oder Schwefelkohlenstoff befeuchtet werden, um die Einwirkung zu märsigen. Nach eintägigem Erhitzen im Wasserbad wurde die Röhre geöffnet und ihr schwach gelb gefärbter Inhalt mit sehr viel Aether wiederholt ausgezogen. Aus 
Wehrhane u. Hühner, iher den Cyanphosphor. 289

diesem schieden sich über Schwefelsäure gelbe, ganz kleine Krystalle ab. Zur Analyse konnte diese Verbindung nicht hinreichend rein erhalten werden, da sich kein starkes Lösungsmittel für sie fand und sio nicht flüchtig war, sondern beim Erhitzen ein stinkendes Oel (vielleicht ES, abschied.

Auch Antimonchlorid und Cyansilber wurden verschlossen auf $150^{\circ}$ erhitzt. Beim Oefinen des Verschlusses zeigte sich Druck und der Geruch des Chlorcyans, während die Wände des Gefäfses mit cyanfreien Krystallen bekleidet waren. Das Antimonchlorid scheint daher zu einem geringen Theil in Antimonoblorūr unter Bildung von Chlorcyan und Chlorsilber zerfallen zu sein. Ein Cyanantimon konnte nicht abgeschieden werden; gegen das Vorhandenscin einer solchea Verbindung spricht auch der Versuch von Gay-Lussac*), welcher beim Ueberleiten von Chlorcyangas über erhitztes Antimon Chlorantimon und freies Cyangas erhielt.

Ebenso konnten entsprechende Mengen Arsenchlorid and Cyansilber ohne Erwärmung gemischt werden und selbst nach langem Erhitzen war keine Umsetzung zu beobachten.

Binige Bemerkungen über die Einwirkung ron. Boz- und Siliciumchlorid auf Cyansilber behaiten wir uns vor.

Laboratorium in Göttingen, den 26. Juni 1864.

Beiträge zur Keuntnifs der Kohlentheerfarbstoffe : II) Phenyltolylamin;
von A. W. Hofmann **).

Die Auffindung des Diphenylamins unter den Zersetzunggproduoten des Anilin-Blau's (Triphenyl-Rosanilin), welche

† Gilb. Ann. LIII, 168.

*) Aus den Monatsberichten der Königl. Academio der Wissenschaften za Berlin mitgetheilt. 